\title{
Resolution of Traumatic Pneumatocele Due to Penetrating Thoracic Trauma
}

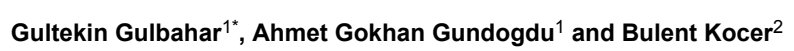

${ }^{1}$ Nafiz Korez Sincan State Hospital, Division of Thoracic Surgery, Ankara Turkey

2 Numune Teaching and Research Hospital, Division of Thoracic Surgery, Ankara Turkey

*Corresponding author: Gultekin Gulbahar, Yasamkent mh Besa Karina sitesi, Yenimahalle/ANKARA, Turkey,Tel:+90505 3359531 ; Fax: +90 312 272 4949 ; E-mail: drbkocer@gmail.com

Rec date: Feb 24, 2014, Acc date: April 15, 2014, Pub date: April 17, 2014

Copyright: @ 2014 Gulbahar G, This is an open-access article distributed under the terms of the Creative Commons Attribution License, which permits unrestricted use, distribution, and reproduction in any medium, provided the original author and source are credited.

\begin{abstract}
Traumatic pneumatocele is rare. It usually regresses spontaneously with conservative treatment. In rare cases, it can get complicated and surgical intervention may be necessary. A 21 year old male patient was taken to the hospital following a fall on an object with a sharp tip. His vital signs were stable. On X-ray and chest-CT he had a pneumatocele area including the injury tract and a low percentage pneumothorax in the right lung. The patient was hospitalized for follow-up and was treated conservatively. After 3 days, he was discharged without any complication.
\end{abstract}

Keywords: Traumatic pneumatocele; Chest-CT; Cavitary lesion; Hemithorax

\section{Introduction}

Traumatic Pneumatocele (TP) is an uncommon cavitary lesion which develops on the pulmonary contusion area following blunt thoracic trauma. Such cavities are named differently according to different authors in English literature. It is especially very rare following penetrating injury and generally treated conservatively. We aimed to discuss briefly the terminology and emphasise the importance of conservative treatment in this study.

\section{Case}

A 21 year old male patient was taken to our hospital following a fall on an object with a sharp tip. There was a chest wall defect reaching the pleural space on the posterolateral aspect of the right hemithorax. He had no complaint other than chest pain. His arterial blood pressure was $110 / 70 \mathrm{mmHg}$; pulse rate was $100 / \mathrm{min}$; respiratory rate was $18 / \mathrm{min}$ and the oxygen saturation was $98 \%$. On chest X-ray he had a lesion consistent with traumatic pneumatocele in the right lung (Figure 1). On the axial computerized tomography of the chest, he had a pneumatocele area including the injury tract and a low percentage pneumothorax (Figure 2). Debridement was done on the chest wall defect and it was sutured primarily. The patient was hospitalized for follow-up and was treated conservatively without an intervention. After 3 days, he was discharged for outpatient clinic follow-up since there was no progression in the pneumothorax and no other complication arised. After 2 weeks, the patient had a almost complete recovery on the chest X-ray (Figure 3).



Figure 1: Chest X-ray of the patient on admission 


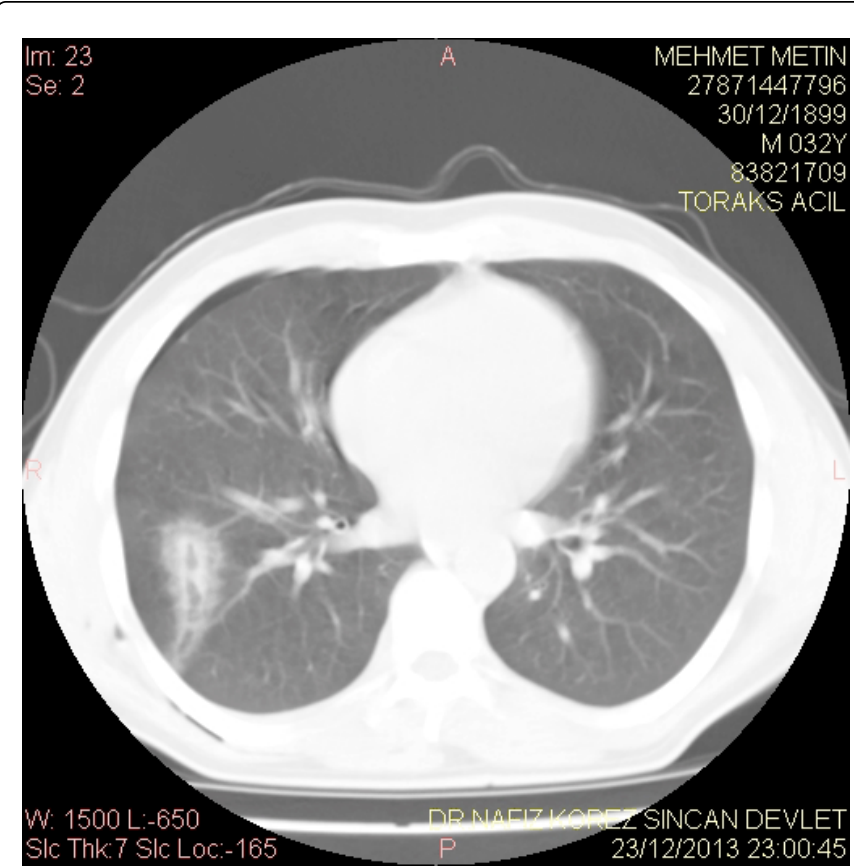

Figure 2: Tract of the stab wound on CT thorax

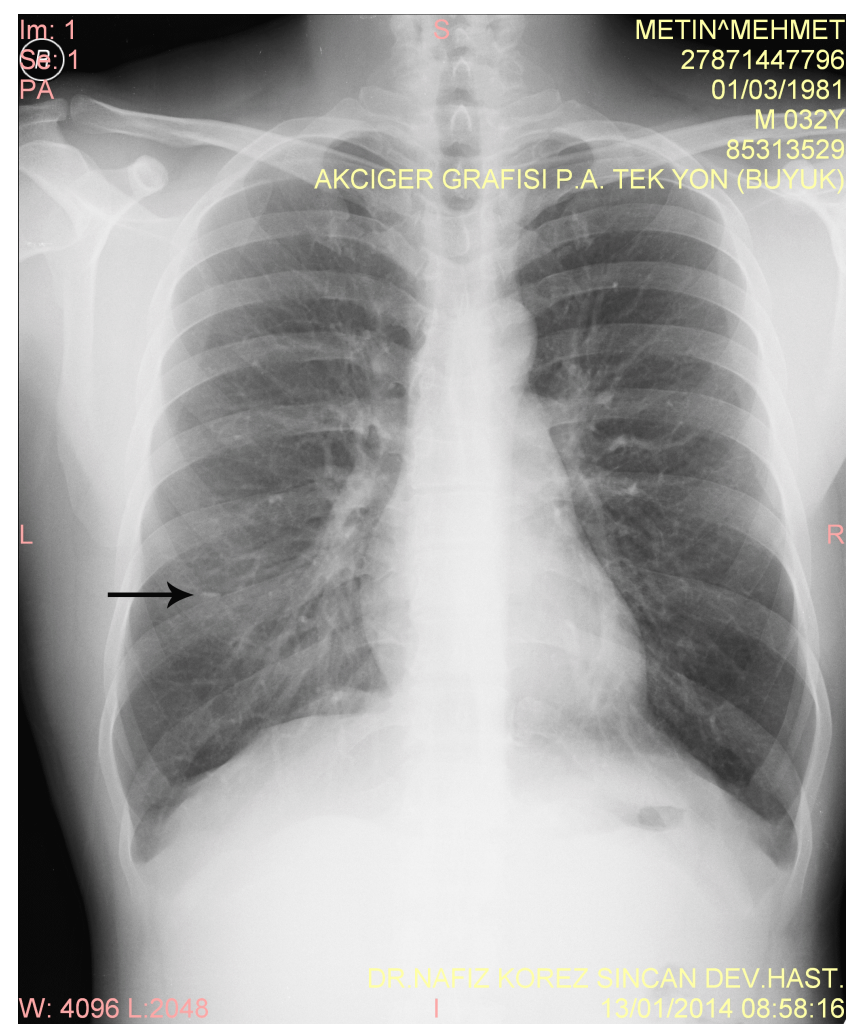

Figure 3: Chest X-ray of the patient after 2 weeks. TPP resolved

\section{Discussion}

"Hematoma", "cyst", "pesudocyst", "pneumatocele" terms have been used in the literature for the radiologic appearances caused by filling of air and/or blood in the parenchymal defect which occurs after trauma. Because of the lacking epithelial lining "traumatic pulmonary pseudocyst (TPP)" term has been suggested instead of "cyst". In another study the lesion is classified as "complicated TPP" and "uncomplicated TPP" according to the blood presence inside the cavity [1,2]. "Pulmonary hematoma", "traumatic pulmonary pseudocyst" and "traumatic pneumatocele" defines such lesion as parenchyma originated blood and/or air fills at various amounts in the parenchymal defect formed after a penetrating or blunt trauma. We believe that such lesions shall be collected under a common heading as "traumatic pulmonary cavitary lesions". Because they have the same etiology; the same kind of parenchymal cells form the lining of the cystic cavities and also the clinical presentation and the treatment modalities are similar. Thus we defined present case as TP since the traumatic pulmonary cavity contains only air.

TP due to blunt thoracic trauma is encountered more commonly in children and young adults because of the elasticity of the thoracic wall. The mechanism which plays role in the development of TP due to blunt trauma is an intraparenchymal pulmonary laceration with airway disruption, air leak and/or filling of blood into the pulmonary parenchyma [3].

$\mathrm{TP}$ due to penetrating trauma is very rare and it may occur at any age. It occurs when air enters lacerated parenchyma and cannot escape to the pleural space as a result of one way or check valve mechanism [4].

Although X-ray may be used in diagnosis; in a study, which mentions 10 TPP cases in a 9 year period, none of the cases were detected on the first day by X-ray, but all were demonstrated on CT [5]. Likewise, in another study of the 12 TPP cases, only 1 was diagnosed by X-ray and all others were diagnosed by CT [2]. It seems that the contusion and the parenchymal edema caused by the blunt trauma makes it hard for the cavitary lesions formed on contusion to be detected by X-ray. However, it is not hard to detect TPP due to penetrating trauma by $\mathrm{x}$-ray. The diagnosis for the case presented herein, was made by X-ray on the first and it was later on confirmed by CT.

The symptoms like chest pain, hemoptysis and cough in these patients, are related to the pulmonary parenchymal damage but not to the TPP. Moreover the cases may be asymptomatic. Hemoptysis is usually not excessive to be life-threatening but sometimes massive hemoptysis requiring lobectomy may be encountered $[5,6]$. The difference between the symptoms of TPP due to blunt and penetrating trauma is related to difference in the amount of accompanying injury to the chest wall and the pulmonary parenchyma and of course this is not surprising.

Kesieme et al. report a case who sustained blunt chest trauma with a huge, tense traumatic pneumatocele which lead to cerebral infarction [7]. But usually TPs resolve spontaneously without complication.

\section{Conclusion}

"Traumatic pulmonary parenchymal cavitary lesions" can be named as "pulmonary hematoma", "traumatic pulmonary pseudocyst" and "traumatic pneumatocele" according to the filling of the cavity with 
Citation: Gulbahar G, Gundogdu AG, Kocer B (2014) Resolution of Traumatic Pneumatocele Due to Penetrating Thoracic Trauma. J Trauma Treat 3: 194. doi:10.4172/2167-1222.1000194

Page 3 of 3

parenchymal blood and/or air. X-ray may be insufficient in diagnosis for blunt trauma cases. In such cases CT examination is necessary.

\section{References}

1. Santos GH, Mahendra T (1979) Traumatic pulmonary pseudocysts. Ann Thorac Surg 27: 359-362.

2. Chon SH, Lee CB, Kim H, Chung WS, Kim YH (2006) Diagnosis and prognosis of traumatic pulmonary psuedocysts: a review of 12 cases. Eur J Cardiothorac Surg 29: 819-823.

3. Tomlanovich MC (1983) Pulmonary parenchymal injuries. Emerg Med Clin North Am 1: 379-392.
4. Kocer B, Gulbahar G, Gunal N, Dural K, Sakinci U (2007) Traumatic pulmonary pseuodocysts: two case reports. J Med Case Rep 1: 112.

5. Melloni G, Cremona G, Ciriaco P, Pansera M, Carretta A, et al. (2003) Diagnosis and treatment of traumatic pulmonary pseudocysts. J Trauma 54: 737-743.

6. Athanassiadi K, Gerazounis M, Kalantzi N, Kazakidis P, Fakou A, et al. (2003) Primary traumatic pulmonary pseudocysts: a rare entity. Eur J Cardiothorac Surg 23: 43-45.

7. Kesieme E, Feldmann M, Welcker K, Linder A, Prisadov G (2012) Cerebral infarct complicating traumatic pneumatocele: a rare sequela following blunt chest trauma. Thorac Cardiovasc Surg 60: e16-18. 